\title{
KULEUVEN
}

\begin{tabular}{|l|l|}
\hline Citation & $\begin{array}{l}\text { T.D. Son, G. Pipeleers, J. Swevers (2015), } \\
\text { Robust Monotonic Convergent Iterative Learning Control } \\
\text { IEEE Transactions on Automatic Control }\end{array}$ \\
\hline Archived version & $\begin{array}{l}\text { Author manuscript: the content is identical to the content of the pub- } \\
\text { lished paper, but without the final typesetting by the publisher }\end{array}$ \\
\hline Published version & http://ieeexplore.ieee.org/xpl/freeabs_all.jsp?arnumber=7160689 \\
\hline Publisher homepage & http://ieeexplore.ieee.org \\
\hline Author contact & $\begin{array}{l}\text { E-mail: tong.duyson@kuleuven.be } \\
\text { Phone number: }+32(0) 16372775\end{array}$ \\
\hline IR & \\
\hline
\end{tabular}

(article begins on next page) 


\section{Robust Monotonic Convergent Iterative Learning Control}

Tong Duy Son, Goele Pipeleers and Jan Swevers

\begin{abstract}
This paper presents an approach to deal with model uncertainty in iterative learning control (ILC). Model uncertainty generally degrades the performance of conventional learning algorithms. To deal with this problem, a robust worst-case norm-optimal ILC design is introduced. The design problem is reformulated as a convex optimization problem, which can be solved efficiently. The paper also shows that the proposed robust ILC is equivalent to conventional norm-optimal ILC with trial-varying parameters; accordingly, the design trade-off between robustness and convergence speed is analyzed.
\end{abstract}

\section{INTRODUCTION}

Iterative learning control (ILC) is widely used in control applications to improve performance of repetitive processes [1]. The key idea of ILC is to update the control signal iteratively based on measured data from previous trials, such that the output converges to the given reference trajectory. Most ILC update laws use the system model as a basis for the learning algorithm and the convergence analysis. Since system models are never perfect in practical applications, accounting for model uncertainty in the ILC design needs to be addressed.

The robustness of a variety of ILC approaches has been analyzed in the literature for inverse model-based ILC [2], norm-optimal ILC [3], [4], two dimensional learning systems [5], and gradient-based ILC [6]. In general, these papers derive robust convergence conditions. Some papers present ILC designs that explicitly account for model uncertainty to improve robust convergence and performance. In [7], the authors consider higher order ILC, while [8] investigates the use of time-varying filtering and [9] develops an averaging technique over uncertain models for a robustly converging algorithm. [10] designs a robust ILC that account for interval uncertainty on each impulse response parameter of the lifted system representation, which results in a large implementation effort. Since $\mathcal{H}_{\infty}$ approach based design techniques are a common robust feedback control design, they have also been exploited to design robust ILCs [11], [12].

In this paper, we propose a robust norm-optimal ILC design that takes into account model uncertainty. In order to derive the uncertainty description in the lifted system representation, we first consider the nominal plant, uncertainty weight and unstructured uncertainty models in the frequency domain representation [13]. Then these models are converted into lifted models. The robust ILC design is formulated as a min-max problem in which the worstcase value of a quadratic cost function under model uncertainty is minimized. Accordingly, the robust ILC problem is reformulated as a convex optimization problem such that a globally optimal solution is guaranteed. This work is different from [14] and [15], which have established robust worst-case ILC algorithms: the former considers parametric uncertainty, while the latter is based on $\mathcal{H}_{\infty}$ control theory.

As an additional contribution of the paper, the equivalence between the solution of the proposed robust ILC design and conventional norm-optimal ILC with trial-varying weight matrices is discussed. Even though some works highlight the importance of weight matrices in the convergence speed and converged performance of normoptimal ILC [15], [16], they only consider fixed gains for all trials. Here, we demonstrate the change of weights trial-by-trial in order to achieve robustness, which also provides more insight into the effects

T. D. Son, G. Pipeleers and J. Swevers are with the Department of Mechanical Engineering, Katholieke Universiteit Leuven, Celestijnenlaan 300B, B3001 Heverlee, Belgium. Email: tong. duyson@mech. kuleuven. be. of the weight matrices on robustness and convergence speed of normoptimal ILC.

The remainder of this paper is organized as follows. Section II provides the background on norm-optimal ILC and presents the robust ILC problem. Section III formulates the developed robust ILC design approach, and Section IV shows the equivalence between the robust ILC and classical norm-optimal ILC. Simulation results are given in Section V, and Section VI concludes this paper.

\section{Problem Formulation}

\section{A. System representation}

The ILC design is considered in discrete time, where the discrete time instants are labeled by $k=0,1, \ldots$ and $q$ denotes the forward time shift operator. The trials are labeled by the subscript $j=0,1, \ldots$ Each trial comprises $N$ time samples and prior to each trial the plant is returned to the same initial conditions. The robust ILC design considers linear time-invariant (LTI), single-input single-output (SISO) systems that are subject to unstructured additive uncertainty. That is, the method accounts for a set of systems $P_{\Delta}(q)$ of the following form:

$$
P_{\Delta}(q)=\hat{P}(q)+\Delta(q) W(q), \quad \Delta(q) \in \mathcal{B}_{\Delta},
$$

with

$$
\mathcal{B}_{\Delta}=\left\{\Delta(q)=\text { stable, causal LTI system : }\|\Delta(q)\|_{\infty} \leq 1\right\},
$$

where $\|\cdot\|_{\infty}$ is the $\mathcal{H}_{\infty}$ norm. $\hat{P}(q)$ is the nominal plant model and the weight $W(q)$ determines the size of the uncertainty. $\hat{P}(q)$, $W(q)$, and $\Delta(q)$ are stable transfer functions. Without loss of generality, $\hat{P}(q)$ and $W(q)$ are assumed to have relative degree 1 , and $\Delta(q)$ has relative degree 0 . Let $\hat{p}(k), \delta(k)$ and $w(k)$ denote the impulse responses of $\hat{P}(q), \Delta(q)$ and $W(q)$, respectively, leading to $\hat{P}(q)=\sum_{k=1}^{N} \hat{p}(k) q^{-k}, \Delta(q)=\sum_{k=0}^{N-1} \delta(k) q^{-k}$, and $W(q)=\sum_{k=1}^{N} w(k) q^{-k}$. The system input in trial $j$ is denoted by $u_{j}(k)$, and $y_{j}(k)$ is the system output.

The ILC design is formulated in the trial domain, relying on the lifted system representation [1]. The input and output samples during the trial are grouped into large vectors

$$
\begin{aligned}
\mathbf{u}_{j} & =\left[\begin{array}{llll}
u_{j}(0) & u_{j}(1) & \cdots & u_{j}(N-1)
\end{array}\right]^{T}, \\
\mathbf{y}_{j} & =\left[\begin{array}{llll}
y_{j}(1) & y_{j}(2) & \cdots & y_{j}(N)
\end{array}\right]^{T},
\end{aligned}
$$

and the plant dynamics are reformulated between $\mathbf{u}_{j}$ and $\mathbf{y}_{j}$ :

$$
\mathbf{y}_{j}=\mathbf{P}_{\Delta} \mathbf{u}_{j} .
$$

Let $T$ be the Toeplitz operator:

$$
T\left(x_{1}, x_{2}, \ldots, x_{N}\right):=\left[\begin{array}{cccc}
x_{1} & 0 & \cdots & 0 \\
x_{2} & x_{1} & \ddots & \vdots \\
\vdots & \ddots & \ddots & 0 \\
x_{N} & \cdots & x_{2} & x_{1}
\end{array}\right],
$$

then $\mathbf{P}_{\boldsymbol{\Delta}}$ is given by $\mathbf{P}_{\boldsymbol{\Delta}}=\hat{\mathbf{P}}+\Delta \mathbf{W}$, where

$$
\begin{aligned}
\hat{\mathbf{P}} & =T(\hat{p}(1), \hat{p}(2), \ldots, \hat{p}(N)), \\
\mathbf{W} & =T(w(1), w(2), \ldots, w(N)), \\
\boldsymbol{\Delta} & =T(\delta(0), \delta(1), \ldots, \delta(N-1)) .
\end{aligned}
$$

In the lifted form, the set $\mathcal{B}_{\Delta}$ translates into the following set $\mathcal{B}_{\Delta}$ for the matrices $\Delta$ :

$$
\begin{aligned}
\mathcal{B}_{\boldsymbol{\Delta}}=\{\boldsymbol{\Delta} & =T(\delta(0), \ldots, \delta(N-1)): \Delta(q)=\sum_{k=0}^{\infty} \delta(k) q^{-k} \\
& \left.=\text { stable, causal LTI system with }\|\Delta(q)\|_{\infty} \leq 1\right\} .
\end{aligned}
$$




\section{B. Classical norm-optimal ILC}

In norm-optimal ILC, the control signal is computed by minimizing the following cost function with respect to $\mathbf{u}_{j+1}$ [16], [18]:

$$
J\left(\mathbf{u}_{j+1}, \boldsymbol{\Delta}\right)=\left\|\mathbf{e}_{j+1}\right\|_{\mathbf{Q}}^{2}+\left\|\mathbf{u}_{j+1}-\mathbf{u}_{j}\right\|_{\mathbf{R}}^{2}+\left\|\mathbf{u}_{j+1}\right\|_{\mathbf{S}}^{2},
$$

where $\mathbf{Q}, \mathbf{R}, \mathbf{S}$ are symmetric positive definite matrices. We define $\|x\|^{2}=x^{T} x$ and $\|x\|_{\mathrm{M}}^{2}=x^{T} \mathrm{M} x$. In the cost function, $\mathbf{e}_{j+1}$ is the $(j+1)$-th trial's tracking error, $\mathbf{e}_{j+1}=\mathbf{y}_{d}-\mathbf{P}_{\Delta} \mathbf{u}_{j+1}$ with reference signal $\mathbf{y}_{d}$, then

$$
\begin{aligned}
\mathbf{e}_{j+1} & =\mathbf{e}_{j}-(\hat{\mathbf{P}}+\Delta \mathbf{W})\left(\mathbf{u}_{j+1}-\mathbf{u}_{j}\right) \\
& =\hat{\mathbf{e}}_{j+1}-\Delta \mathbf{W}\left(\mathbf{u}_{j+1}-\mathbf{u}_{j}\right)
\end{aligned}
$$

where $\hat{\mathbf{e}}_{j+1}=\mathbf{e}_{j}-\hat{\mathbf{P}}\left(\mathbf{u}_{j+1}-\mathbf{u}_{j}\right)$.

In classical norm-optimal ILC, the error $\mathbf{e}_{j+1}$ is replaced by the nominal estimated error $\hat{\mathbf{e}}_{j+1}$, assuming $\boldsymbol{\Delta}=0$. This leads to the following ILC update law:

$$
\mathbf{u}_{j+1}=\mathcal{Q} \mathbf{u}_{j}+\mathcal{L} \mathbf{e}_{j}
$$

where

$$
\begin{aligned}
\mathcal{Q} & =\left(\hat{\mathbf{P}}^{T} \mathbf{Q} \hat{\mathbf{P}}+\mathbf{S}+\mathbf{R}\right)^{-1}\left(\hat{\mathbf{P}}^{T} \mathbf{Q} \hat{\mathbf{P}}+\mathbf{R}\right), \\
\mathcal{L} & =\left(\hat{\mathbf{P}}^{T} \mathbf{Q} \hat{\mathbf{P}}+\mathbf{S}+\mathbf{R}\right)^{-1} \hat{\mathbf{P}}^{T} \mathbf{Q} .
\end{aligned}
$$

1) Nominal monotonic convergence: The ILC algorithm (6) is nominal monotonic convergent if $\|\mathcal{Q}-\mathcal{L} \hat{\mathbf{P}}\|<1$ [1], yielding

$$
\left\|\left(\hat{\mathbf{P}}^{T} \mathbf{Q} \hat{\mathbf{P}}+\mathbf{S}+\mathbf{R}\right)^{-1} \mathbf{R}\right\|<1 .
$$

2) Robust monotonic convergence: The ILC algorithm (6) is robust monotonic convergent if $\left\|\mathcal{Q}-\mathcal{L P}_{\Delta}\right\|<1$ for all $\boldsymbol{\Delta} \in \mathcal{B}_{\boldsymbol{\Delta}}$ [15], [16], yielding

$$
\left\|\left(\hat{\mathbf{P}}^{T} \mathbf{Q} \hat{\mathbf{P}}+\mathbf{S}+\mathbf{R}\right)^{-1}\left(\mathbf{R}-\hat{\mathbf{P}}^{T} \mathbf{Q} \boldsymbol{\Delta} \mathbf{W}\right)\right\|<1, \quad \forall \boldsymbol{\Delta} \in \mathcal{B}_{\boldsymbol{\Delta}} .
$$

Some attempts to remove $\boldsymbol{\Delta}$ for deriving a robust monotonic convergence condition usually results in conservative results [3], [8], [16]. Satisfying the nominal monotonic convergence (7) does not necessarily imply robust monotonic convergence. A common approach to satisfy (8) is to increase $\mathbf{S}$ but this then reduces the converged performance [18]. This compromise motivates our robust ILC design approach such that both monotonic convergence and high performance are achieved.

\section{Robust norm-optimal ILC}

In this work, we minimize the cost function (4) without the assumption $\boldsymbol{\Delta}=0$. Instead, we propose a robust norm-optimal ILC design by considering the following worst-case optimization problem:

$$
\underset{\mathbf{u}_{j+1}}{\operatorname{minimize}} \sup _{\boldsymbol{\Delta} \in \mathcal{B}_{\boldsymbol{\Delta}}}\left\{J\left(\mathbf{u}_{j+1}, \boldsymbol{\Delta}\right)\right\}
$$

where substituting (5) in (4) yields

$$
\begin{aligned}
J\left(\mathbf{u}_{j+1}, \boldsymbol{\Delta}\right)=\left\|\hat{\mathbf{e}}_{j+1}-\Delta \mathbf{W}\left(\mathbf{u}_{j+1}-\mathbf{u}_{j}\right)\right\|_{\mathbf{Q}}^{2} \\
+\left\|\mathbf{u}_{j+1}-\mathbf{u}_{j}\right\|_{\mathbf{R}}^{2}+\left\|\mathbf{u}_{j+1}\right\|_{\mathbf{S}}^{2} .
\end{aligned}
$$

\section{RoBUST ILC DESIGN}

This section presents the proposed robust ILC algorithm, and consequently, analyzes its convergence. First, to obtain a tractable reformulation of the robust ILC design, the set $\mathcal{B}_{\Delta}$ is replaced by an outer approximation:

$$
\mathcal{B}_{\Delta}^{\mathrm{o}}=\left\{\boldsymbol{\Delta} \in \mathbb{R}^{N \times N}:\|\boldsymbol{\Delta}\|<1\right\},
$$

where $\|$.$\| is the induced matrix 2-norm. Hence, we replace$ $\|\Delta(q)\|_{\infty} \leq 1$ by $\|\Delta\|<1$, and extend the set of lower triangular
Toeplitz matrices to $\mathbb{R}^{N \times N}$. With the first replacement, we also extent the set $\mathcal{B}_{\Delta}$ since for stable, causal, LTI systems $\Delta(q)$, it holds that $\|\boldsymbol{\Delta}\| \leq\|\Delta(q)\|_{\infty}$ [8], [17]. In addition, equality holds for $N \rightarrow \infty$. This replacement yields the following robust optimization problem:

$$
\underset{\mathbf{u}_{j+1}}{\operatorname{minimize}} \sup _{\boldsymbol{\Delta} \in \mathcal{B}_{\boldsymbol{\Delta}}^{\circ}}\left\{J\left(\mathbf{u}_{j+1}, \boldsymbol{\Delta}\right)\right\} .
$$

As $\mathcal{B}_{\Delta} \subset \mathcal{B}_{\Delta}^{\circ}$, a solution of (11) is feasible to (9), although generally not optimal.

\section{A. Robust ILC Algorithm}

The following theorem presents the solution of the proposed robust ILC problem (11), which is the main contribution of this work.

Theorem 3.1: The robust ILC problem (11) is equivalent to the following optimization problem,

$$
\begin{array}{ll}
\underset{\mathbf{u}_{j+1}, \lambda_{j+1}}{\operatorname{minimize}} & J_{\text {dual }}\left(\mathbf{u}_{j+1}, \lambda_{j+1}\right) \\
\text { subject to } & \lambda_{j+1} \mathbf{I}-\mathbf{Q} \succeq 0 \\
& \mathbf{Q} \hat{\mathbf{e}}_{j+1} \in \mathcal{R}\left(\mathbf{Q}-\lambda_{j+1} \mathbf{I}\right),
\end{array}
$$

where $J_{\text {dual }}\left(\mathbf{u}_{j+1}, \lambda_{j+1}\right)$ denotes the dual cost function,

$$
\begin{aligned}
& J_{\text {dual }}\left(\mathbf{u}_{j+1}, \lambda_{j+1}\right)=\hat{\mathbf{e}}_{j+1}^{T}\left(\mathbf{Q}^{-1}-\lambda_{j+1}^{-1} \mathbf{I}\right)^{\dagger} \hat{\mathbf{e}}_{j+1} \\
& +\lambda_{j+1}\left\|\mathbf{W}\left(\mathbf{u}_{j+1}-\mathbf{u}_{j}\right)\right\|^{2}+\left\|\mathbf{u}_{j+1}-\mathbf{u}_{j}\right\|_{\mathbf{R}}^{2}+\left\|\mathbf{u}_{j+1}\right\|_{\mathbf{S}}^{2},
\end{aligned}
$$

and $\lambda_{j+1}$ is a scalar variable. $\mathcal{R}(A)$ is the range of $A$ and $A^{\dagger}$ is the pseudo-inverse of $A$.

Proof: See Appendix A. Furthermore, (12) can be transformed into a convex optimization problem, as shown in Remark 3.2.

Remark 3.1: Since $\mathbf{Q}$ is a positive definite matrix and $\lambda_{j+1} \mathbf{I}-\mathbf{Q} \succeq$ 0 , the optimal solution of $\lambda_{j+1}$ is guaranteed to be strictly positive.

Remark 3.2: Using the Schur complement with a slack variable $t \in \mathbb{R}$ and $\alpha_{j+1}=\lambda_{j+1}^{-1}$, the robust ILC problem (12) can be found equivalent to the following convex semidefinite program (SDP) [19]:

$$
\begin{array}{ll}
\underset{t, \mathbf{u}_{j+1}, \alpha_{j+1}}{\operatorname{minimize}} & \tilde{J}_{\text {dual }}\left(\mathbf{u}_{j+1}, \alpha_{j+1}, t\right) \\
\text { subject to } & {\left[\begin{array}{cc}
\mathbf{Q}^{-1}-\alpha_{j+1} \mathbf{I} & \hat{\mathbf{e}}_{j+1} \\
\hat{\mathbf{e}}_{j+1}^{T} & t
\end{array}\right] \succeq 0,}
\end{array}
$$

where

$$
\begin{aligned}
\tilde{J}_{\text {dual }}\left(\mathbf{u}_{j+1}, \alpha_{j+1}, t\right)=t & +\alpha_{j+1}^{-1}\left\|\mathbf{W}\left(\mathbf{u}_{j+1}-\mathbf{u}_{j}\right)\right\|^{2} \\
& +\left\|\mathbf{u}_{j+1}-\mathbf{u}_{j}\right\|_{\mathbf{R}}^{2}+\left\|\mathbf{u}_{j+1}\right\|_{\mathbf{S}}^{2} .
\end{aligned}
$$

Note that $\alpha_{j+1}^{-1}\left\|\mathbf{W}\left(\mathbf{u}_{j+1}-\mathbf{u}_{j}\right)\right\|^{2}$ is a quadratic over linear function, which is convex [19].

Remark 3.3: Input constraints such as $\left\|\mathbf{u}_{j+1}\right\|_{\infty} \leq \bar{u}$ and $\left\|\boldsymbol{\delta} \boldsymbol{u}_{j+1}\right\|_{\infty} \leq \overline{\delta u}$, where $\delta u_{j+1}(k)=u_{j+1}(k)-u_{j+1}(k-1)$, can be added in the proposed robust ILC design (12) and (13) without destroying the convexity of the problem. Notice that when the input constraints are imposed, the weight $\mathbf{S}$ on input energy in the cost function may be put equal to zero.

Corollary 3.1: Consider the special case of weight matrix: $\mathbf{Q}=$ $\bar{q} \mathbf{I}$. The robust problem is then simplified since the worst-case cost function $J_{\mathrm{wc}}\left(\mathbf{u}_{j+1}\right)$ with respective to model uncertainty $\boldsymbol{\Delta}$ in (10), $J_{\mathrm{wc}}\left(\mathbf{u}_{j+1}\right)=\sup _{\|\boldsymbol{\Delta}\| \leq 1} J\left(\mathbf{u}_{j+1}, \boldsymbol{\Delta}\right)$, can now be computed explicitly as a consequence of triangle inequality, i.e.

$$
\begin{aligned}
& J_{\mathrm{wc}}\left(\mathbf{u}_{j+1}\right)=\bar{q}\left(\left\|\hat{\mathbf{e}}_{j+1}\right\|+\left\|\mathbf{W}\left(\mathbf{u}_{j+1}-\mathbf{u}_{j}\right)\right\|\right)^{2} \\
&+\left\|\mathbf{u}_{j+1}-\mathbf{u}_{j}\right\|_{\mathbf{R}}^{2}+\left\|\mathbf{u}_{j+1}\right\|_{\mathbf{S}}^{2} .
\end{aligned}
$$

Consequently, the robust problem (11) is now a second order cone program (SOCP),

$$
\underset{\mathbf{u}_{j+1}}{\operatorname{minimize}} J_{\mathrm{wc}}\left(\mathbf{u}_{j+1}\right) .
$$


This can be solved effectively using convex programming [19]. The cost function $J_{\mathrm{wc}}\left(\mathbf{u}_{j+1}\right)$ shows that the convergence speed depends not only on $\mathbf{R}$ but also on the uncertainty weight $\mathbf{W}$. The speed decreases as $\mathbf{W}$ increases. Optimization problem (15) can also be retrieved from (12). Partial minimization of $J_{\text {dual }}$ with respect to $\lambda_{j+1}$ yields

$$
\lambda_{j+1}=\bar{q}\left(1+\frac{\left\|\hat{\mathbf{e}}_{j+1}\right\|}{\left\|\mathbf{W}\left(\mathbf{u}_{j+1}-\mathbf{u}_{j}\right)\right\|}\right),
$$

if $\mathbf{W}\left(\mathbf{u}_{j+1}-\mathbf{u}_{j}\right) \neq 0$, and $\lambda_{j+1}=+\infty$ otherwise. The solution satisfies the constraint $\lambda_{j+1}>\bar{q}$ in (12). Plugging in this solution in $J_{\text {dual }}\left(\mathbf{u}_{j+1}, \lambda_{j+1}\right)$ in (12b) yields $J_{\text {wc }}\left(\mathbf{u}_{j+1}\right)$.

\section{B. Convergence}

This subsection analyzes the convergence of the robust ILC design.

Theorem 3.2: The robust ILC design (12) guarantees the monotonic convergence of the worst-case cost function as

$$
\left\|\mathbf{e}_{j+1}\right\|_{\mathbf{Q}}^{2}+\left\|\mathbf{u}_{j+1}\right\|_{\mathbf{S}}^{2} \leq J_{\mathrm{wc}}\left(\mathbf{u}_{j+1}\right) \leq\left\|\mathbf{e}_{j}\right\|_{\mathbf{Q}}^{2}+\left\|\mathbf{u}_{j}\right\|_{\mathbf{S}}^{2},
$$

and the inequalities are strict unless $\mathbf{u}_{j+1}=\mathbf{u}_{j}$.

Proof: Only the case $\mathbf{Q}=\bar{q} \mathbf{I}$ is considered here. The proof for a general symmetric matrix $\mathbf{Q}$ is similar and presented in Appendix B. Define $\mathbf{u}_{j+1}^{*}$ as the $(j+1)$-th optimal input, then $J_{\mathrm{wc}}\left(\mathbf{u}_{j+1}^{*}\right) \leq$ $\left.J_{\mathrm{wc}}\left(\mathbf{u}_{j+1}\right)\right|_{\mathbf{u}_{j+1}=\mathbf{u}_{j}}$. Hence, replacing $\mathbf{u}_{j+1}$ by $\mathbf{u}_{j}$ in (14) yields

$$
J_{\mathrm{wc}}\left(\mathbf{u}_{j+1}^{*}\right) \leq\left\|\mathbf{e}_{j}\right\|_{\mathbf{Q}}^{2}+\left\|\mathbf{u}_{j}\right\|_{\mathbf{S}}^{2} .
$$

Equality only happens when $\mathbf{u}_{j+1}=\mathbf{u}_{j}$ since (13b) is a strictly convex function with respect to $\mathbf{u}_{j+1}[19]$. Moreover, for $\Delta \in \mathcal{B}_{\Delta}^{\text {o }}$, we have

$$
\left\|\mathbf{e}_{j+1}\right\|_{\mathbf{Q}}^{2}+\left\|\mathbf{u}_{j+1}\right\|_{\mathbf{S}}^{2} \leq J\left(\mathbf{u}_{j+1}, \boldsymbol{\Delta}\right) \leq J_{\mathrm{wc}}\left(\mathbf{u}_{j+1}^{*}\right) .
$$

Since the left inequality follows from subtracting the term $\| \mathbf{u}_{j+1}$ $\mathbf{u}_{j} \|_{\mathbf{R}}^{2}$ in (4), it is strict unless $\mathbf{u}_{j+1}=\mathbf{u}_{j}$. As a result, we obtain the inequalities (17).

Remark 3.4: The relationship (17) shows that when $\mathbf{S}=0$ or there are no constraints on the input signal, the ILC controller can achieve monotonic convergence of the tracking error, i.e. $\left\|\mathbf{e}_{j+1}\right\| \leq\left\|\mathbf{e}_{j}\right\|$.

\section{INTERPRETATION OF THE RESULTS AS AN ADAPTIVE ILC}

This section discusses the equivalence between the developed robust ILC approach and the classical norm-optimal ILC formulation. Furthermore, this relationship is used to analyze convergence speed of the robust algorithm with respect to model uncertainty.

Theorem 4.1: The robust ILC problem (12) is equivalent to the following ILC algorithm,

$$
\mathbf{u}_{j+1}=\mathcal{Q}_{j+1}\left(\lambda_{j+1}\right) \mathbf{u}_{j}+\mathcal{L}_{j+1}\left(\lambda_{j+1}\right) \mathbf{e}_{j},
$$

where

$$
\begin{aligned}
\mathcal{Q}_{j+1}\left(\lambda_{j+1}\right) & =\left(\hat{\mathbf{P}}^{T} \mathbf{Q}_{j+1} \hat{\mathbf{P}}+\mathbf{R}_{j+1}+\mathbf{S}\right)^{-1}\left(\hat{\mathbf{P}}^{T} \mathbf{Q}_{j+1} \hat{\mathbf{P}}+\mathbf{R}_{j+1}\right) \\
\mathcal{L}_{j+1}\left(\lambda_{j+1}\right) & =\left(\hat{\mathbf{P}}^{T} \mathbf{Q}_{j+1} \hat{\mathbf{P}}+\mathbf{R}_{j+1}+\mathbf{S}\right)^{-1} \hat{\mathbf{P}}^{T} \mathbf{Q}_{j+1},
\end{aligned}
$$

where $\mathbf{Q}_{j+1}$ and $\mathbf{R}_{j+1}$ are the weight matrices at the $(j+1)$-th trial, dependent on $\lambda_{j+1}$, and are given by

$$
\begin{aligned}
& \mathbf{Q}_{j+1}\left(\lambda_{j+1}\right)=\left(\mathbf{Q}^{-1}-\lambda_{j+1}^{-1} \mathbf{I}\right)^{\dagger}, \\
& \mathbf{R}_{j+1}\left(\lambda_{j+1}\right)=\mathbf{R}+\lambda_{j+1} \mathbf{W}^{T} \mathbf{W} .
\end{aligned}
$$

Moreover, the solution of $\lambda_{j+1}$ is obtained from the following nonlinear optimization problem:

$$
\begin{array}{ll}
\underset{\lambda_{j+1}}{\operatorname{minimize}} & J_{\text {dual }}\left(\mathbf{u}_{j+1}\left(\lambda_{j+1}\right), \lambda_{j+1}\right) \\
\text { subject to } & \lambda_{j+1} \mathbf{I}-\mathbf{Q} \succeq 0 \\
& \mathbf{Q} \hat{\mathbf{e}}_{j+1} \in \mathcal{R}\left(\mathbf{Q}-\lambda_{j+1} \mathbf{I}\right) .
\end{array}
$$

Proof: We first rewrite the optimization problem (12) as follows

$$
\begin{array}{cl}
\underset{\lambda_{j+1}}{\operatorname{minimize}} \underset{\mathbf{u}_{j+1}}{\operatorname{minimize}} & J_{\text {dual }}\left(\mathbf{u}_{j+1}, \lambda_{j+1}\right) \\
\text { subject to } & \lambda_{j+1} \mathbf{I}-\mathbf{Q} \succeq 0, \\
& \mathbf{Q} \hat{\mathbf{e}}_{j+1} \in \mathcal{R}\left(\mathbf{Q}-\lambda_{j+1} \mathbf{I}\right) .
\end{array}
$$

The minimization problem $\min _{\mathbf{u}_{j+1}} J_{\text {dual }}$ is a convex problem with respect to $\mathbf{u}_{j+1}$, hence the optimal input is achieved by differentiating the cost function with respect to $\mathbf{u}_{j+1}$, yielding (20). The optimal $\lambda_{j+1}$ is found from the optimization problem (22). Once $\lambda_{j+1}$ is calculated, the learning gains in the ILC law (20) are obtained.

Remark 4.1: By comparing of the robust worst-case ILC design described by (20)-(22) with classical ILC (6), the formula is the same except that the learning matrices are updated trial-by-trial. Particularly, the robust design depends on trial-varying $\mathbf{Q}_{j+1}$ and $\mathbf{R}_{j+1}$, while $\mathbf{S}$ remains trial-invariant.

Remark 4.2: As an effect of the convergence of the robust ILC, i.e. $\mathbf{u}_{j+1} \rightarrow \mathbf{u}_{j}$ as $j \rightarrow \infty$, the solution of $\lambda_{j+1}$ shows that $\lambda_{j+1} \rightarrow+\infty$ as $j \rightarrow \infty$. Thus $\mathbf{Q}_{j+1}$ converges to $\mathbf{Q}$, while $\left\|\mathbf{R}_{j+1}\right\|$ increases eventually to a very large value in the trial domain. The learning speed is decreased as the number of trial increases.

Remark 4.3: From (16) and (21), the adaptive ILC interpretation provides additional analyses for the robust ILC algorithm:

- When the amount of uncertainty is very small, i.e. $\|\mathbf{W}\| \approx 0$, the updated weights are approximately equal to the given $\mathbf{Q}$ and $\mathbf{R}$ which means that the robust ILC design is now analogous to classical ILC.

- As discussed in Section III.D, the larger is $\mathbf{W}$, the larger is $\lambda_{j+1}$, resulting in larger $\left\|\mathbf{R}_{j+1}\right\|$ while $\left\|\mathbf{Q}_{j+1}\right\|$ is smaller and closer to $\|\mathbf{Q}\|$. Thus, the convergence speed is slower and the system performance is decreased. This demonstrates the compromise between robustness and convergence speed in the proposed robust ILC design.

Remark 4.4: When $\mathbf{S}=0$, i.e. there are no constraints on the input signal, then $\mathcal{Q}_{j+1}=\mathbf{I}$. As a result of Theorem 3.2, the robust ILC performance achieves perfect asymptotic tracking error, i.e. $\mathbf{e}_{\infty}=0$.

\section{NUMERICAL ILLUSTRATION}

Consider the uncertain plant: $P_{\Delta}(s)=\hat{P}(s)+\Delta(s) W(s)$, where the nominal model:

$$
\hat{P}(s)=\frac{5}{s+1},
$$

and the additive uncertainty weight transfer function:

$$
W(s)=\frac{10 s}{(s+1)(s+6)} .
$$

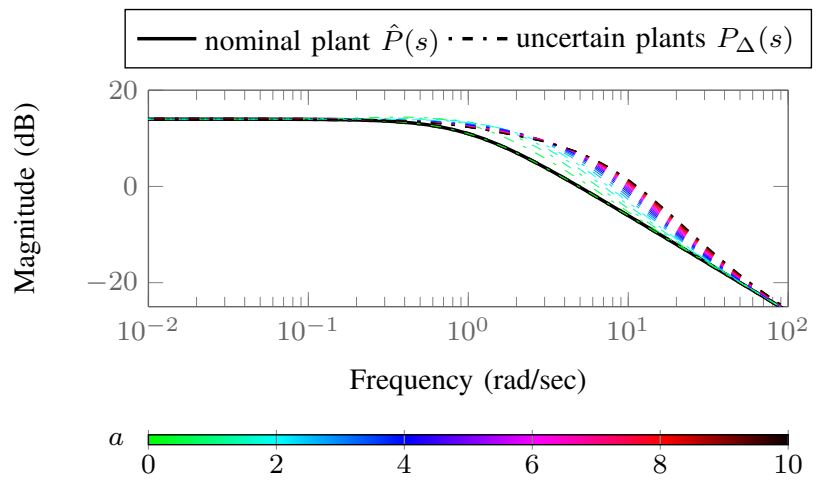

Fig. 1: Bode-diagrams of uncertain plants 


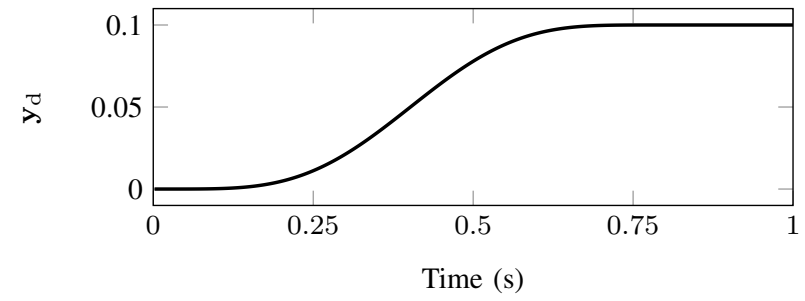

Fig. 2: Reference output

In order to simulate the ILC algorithms with model uncertainty, one set of uncertain models is considered:

$$
\Delta(s)=-\frac{s-a}{s+a} ; \quad a=0, \ldots, 10 .
$$

Fig. 1 shows the Bode plots of the nominal model $\hat{P}(s)$ and the selected uncertain plants $P_{\Delta}(s)$ that account for the considered $\Delta(s)$ (26). Next, the nominal model, uncertainty weight and unstructured uncertainty model are discretized with sampling time $T=0.002 \mathrm{~s}$, then lifted with $N=500$ samples, obtaining the lifted models $\hat{\mathbf{P}}, \mathbf{W}$, and $\Delta$, respectively. Note that $\|\Delta(z)\|_{\infty}=1$ for the uncertainties (26), while the lifted uncertainty models have their 2-norm $\|\Delta\| \lesssim 1$. The reference is a smoothed step function, shown in Fig. 2.

The validation of our robust ILC design comprises three main parts. First, we compare the difference in nominal and worst-case performance after one trial. This analysis over one trial allows comparing our robust ILC approach and classical norm-optimal ILC for the whole considered uncertainty class, not only the particular instances considered in (26). In addition, it provides valuable information with respect to a complete trial domain analysis. Second, complete trial domain analyses are performed. Finally, the performance of the robust and classical ILC approaches are compared when robust monotonic convergence conditions are imposed upon both.

\section{A. One trial analysis}

This part analyzes the effects of the proposed robust ILC and classical norm-optimal ILC on the cost functions after one trial. Without loss of generality, the first trial's input is zero. The learning gains are selected as scaled identity matrices, $(\mathbf{Q}, \mathbf{R}, \mathbf{S})=(1,0.4,0.0001) \mathbf{I}$ for both designs. Note that with this selection, the nominal monotonic convergence condition (7) is satisfied. The second trial's input and error are computed using both approaches.

TABLE I: Second trial's cost

\begin{tabular}{ccc}
\hline & Robust ILC & Classical ILC \\
\hline$\hat{J}$ & $247.56 \cdot 10^{-3}$ & $243.59 \cdot 10^{-3}$ \\
\hline$J_{\mathrm{wc}}$ & $1110.57 \cdot 10^{-3}$ & $1121.22 \cdot 10^{-3}$ \\
\hline
\end{tabular}

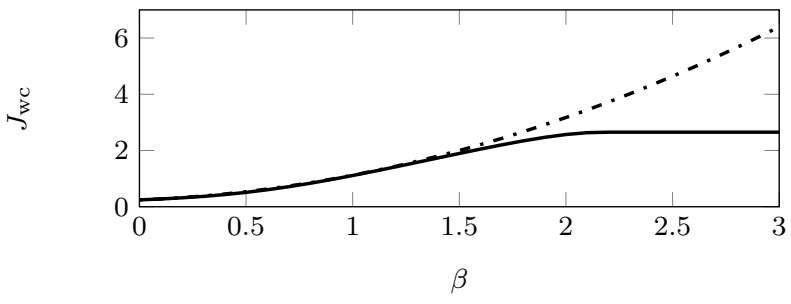

Fig. 3: Worst-case cost with scaling $\mathbf{W}$ of robust ILC (solid) and classical norm-optimal ILC (dashed)

First, the second trial's nominal cost and worst-case cost for all $\boldsymbol{\Delta} \in \mathcal{B}_{\boldsymbol{\Delta}}^{\mathrm{o}}$, denoted by $\hat{J}$ and $J_{\mathrm{wc}}$ respectively, are compared (Table
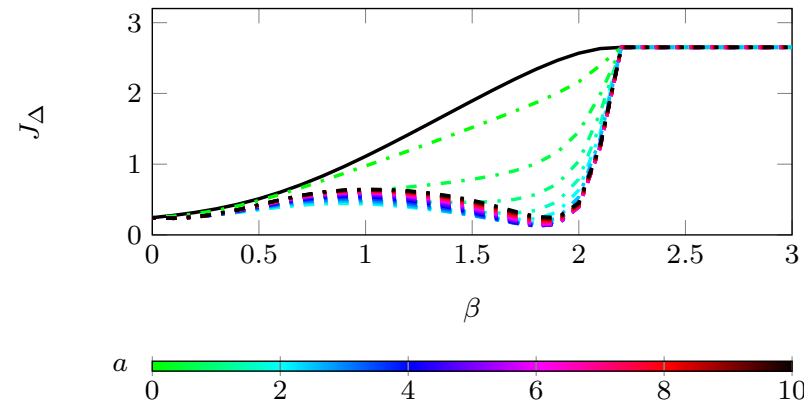

Fig. 4: Robust ILC costs with the uncertain plants $P_{\Delta}(s)$ (dashed) and the worst-case cost (solid) with scaling $\mathbf{W}$

I). The worst-case cost values are calculated using Eq. (14). The table shows that the robust ILC achieves smaller worst-case cost than the classical norm-optimal ILC. We further evaluate the wost-case cost with the set $\Delta \in \mathcal{B}_{\Delta}^{\circ}$ for different uncertainty weights, obtaining by scaling the uncertainty weight as $\beta \mathbf{W}$, where $\beta=0,0.1, \ldots, 3.0$, shown in Fig. 3. The figure shows that the robust ILC has smaller worst-case values than the classical ILC with all scaled $\beta$, and the difference is larger as $\beta$ increases. From $\beta=2.2$, the robust ILC's worst-case cost remains at almost constant values and the updated input is approximately zero, which mean that the robust learning is very slow or even turned off to avoid an excessive cost. In contrast, the classical ILC's worst-case cost keeps increasing.

To gain insight in the conservatism introduced by extending $\mathcal{B}_{\Delta}$ to $\mathcal{B}_{\Delta}^{\circ}$, Fig. 4 shows the worst-case second trial's cost $J_{\mathrm{wc}}$ of the robust ILC approach together with the cost obtained for the particular uncertainty instances of the form (26). Even in this limited set of feasible uncertainties, the worst-case cost $J_{\mathrm{wc}}$ is approached quite closely. This suggests that for the problem at hand, the conservatism introduced in our approach is modest.

\section{B. Trial domain analysis}

This part compares the proposed robust ILC and classical normoptimal ILC performances in the trial domain. The uncertainty weight $\mathbf{W}$ and ILC gains $(\mathbf{Q}, \mathbf{R}, \mathbf{S})$ are selected the same as in the previous subsection. The simulations are conducted using the nominal model $\hat{\mathbf{P}}$ and the model uncertainties given by (26). The tracking errors achieved using the classical ILC and robust ILC are shown in Fig. 5 and Fig. 6, respectively.

From the simulation results in Fig. 5, it can be seen that the classical norm-optimal ILC shows divergence of the tracking error

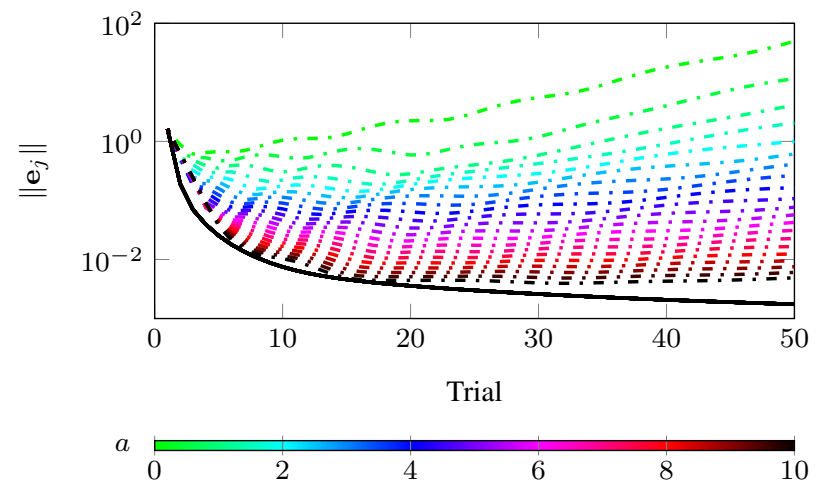

Fig. 5: Performance of classical norm-optimal ILC with nominal plant (solid line) and uncertain plants (dashed lines) 

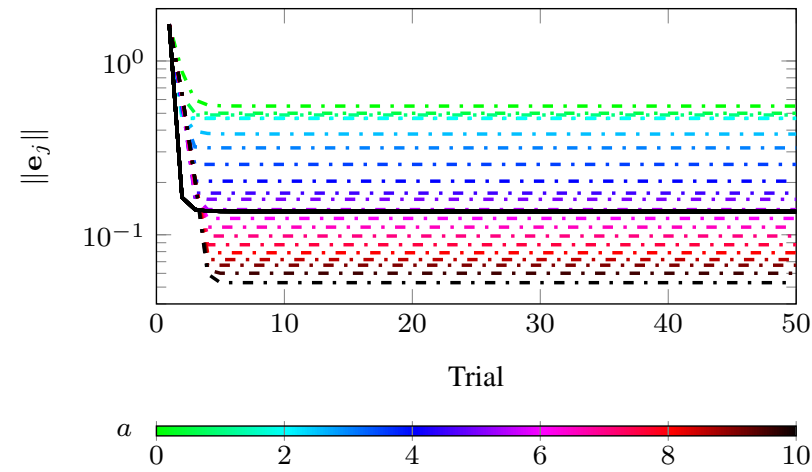

Fig. 6: Performance of robust norm-optimal ILC with nominal plant (solid) and uncertain plants (dashed)

with most uncertain plants even though monotonic convergence for the nominal model is satisfied. In contrast, the proposed robust ILC yields monotonic convergence of the tracking error for the whole uncertainty set (26), shown in Fig. 6. This demonstrates the main advantage of the robust ILC design over the classical ILC: the robust ILC achieves monotonic convergence for all uncertainties $\Delta$. This also confirms that the nominal monotonic convergence condition is not sufficient to obtain convergence for uncertain plants.

Next, we select one uncertain model in (26) with $a=10$ :

$$
\bar{\Delta}(s)=-\frac{s-10}{s+10},
$$

with the corresponding lifted model denoted as $\overline{\boldsymbol{\Delta}}$. Then the worstcase cost $J_{\mathrm{wc}}\left(\mathbf{u}_{j+1}\right)$ is calculated for each trial and compared to $\left\|\mathbf{e}_{j}\right\|_{\mathbf{Q}}^{2}+\left\|\mathbf{u}_{j}\right\|_{\mathbf{S}}^{2}$ with respect to the uncertain model case $\bar{\Delta}$ in order to confirm the inequalities (17) and the convergence of the robust ILC algorithm. This comparison is shown in Fig. 7.

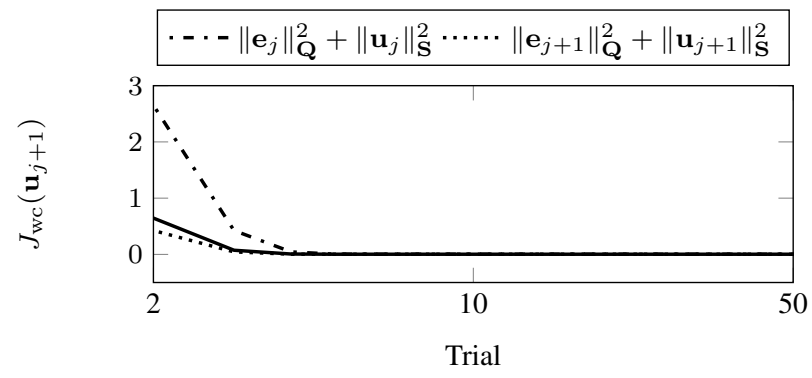

Fig. 7: Convergence of cost function

\section{Trial-varying learning gains}

In the next simulations, we examine the equivalence between the proposed robust ILC design and norm-optimal ILC with trial-varying gains. We use the same uncertain model $\bar{\Delta}(27)$, uncertainty weight $\mathbf{W}$, and learning gains $(\mathbf{Q}, \mathbf{R}, \mathbf{S})$ as in the previous paragraphs. Applying the equivalent adaptive ILC algorithm (20), yields the trial domain variation of $\mathbf{Q}_{j}$ and $\mathbf{R}_{j}$ as shown in Fig. 8 (solid lines). $\left\|\mathbf{Q}_{j}\right\|$ increases in the second trial and then converges to $\|\mathbf{Q}\|$, while $\left\|\mathbf{R}_{j}\right\|$ increases over the trials and then remains at almost constant high values after 6 trials, as discussed in Section IV. Besides, Fig. 7 demonstrates a similar convergence of cost function, that is, the values of the cost hardly change after 6 trials. The updated $\mathbf{Q}_{j}$ and $\mathbf{R}_{j}$ result in a slower convergence than the classical ILC, which can be explained by the fact that the convergence speed is decreased in order to increase the robustness.
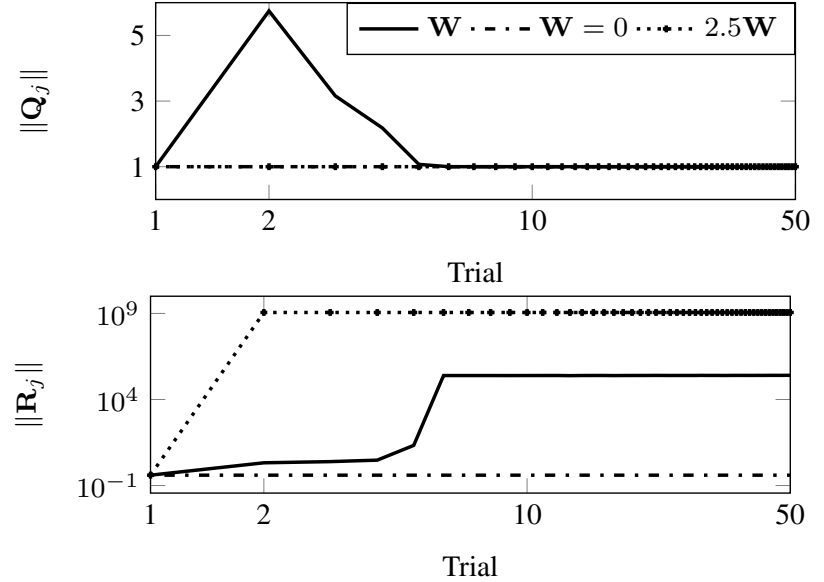

Fig. 8: Trial-varying gains with different uncertainty weights

Fig. 8 also demonstrates the trial-dependency of $\mathbf{Q}_{j}$ and $\mathbf{R}_{j}$ for other uncertainty weights: $\mathbf{W}=0$ and $2.5 \mathbf{W}$. When $\mathbf{W}=0$, the figure shows that $\left\|\mathbf{Q}_{j}\right\|$ and $\left\|\mathbf{R}_{j}\right\|$ remain constant equal to $\|\mathbf{Q}\|$ and $\|\mathbf{R}\|$, respectively. Clearly, when there is no uncertainty in the plant, it is enough to just apply classical norm-optimal ILC. When the weight is $2.5 \mathbf{W},\left\|\mathbf{Q}_{j}\right\|$ jump slightly (not visible) before converging to $\|\mathbf{Q}\|$ while $\left\|\mathbf{R}_{j}\right\|$ increases to a very large value immediately in the second trial, which means that the learning is almost turned off. This analysis confirms the results in Section VI.A, where the robust cost function does not change when the uncertainty weight is larger than $2.2 \mathbf{W}$.

\section{Comparison: Robust ILC vs Classical norm-optimal ILC with robust convergence condition}

The final simulations analyze more in detail the performance of both approaches, especially when the learning gains of the classical ILC are tuned such that the robust monotonic convergence condition is satisfied. But first, the simulations are performed with uncertainty weight $0.5 \mathbf{W}$ and learning gains: $(\mathbf{Q}, \mathbf{R}, \mathbf{S})=\left(1,10^{-5}, 0\right) \mathbf{I}$, which are different from the previous parts. The uncertain model is again given by $\bar{\Delta}$ (27).

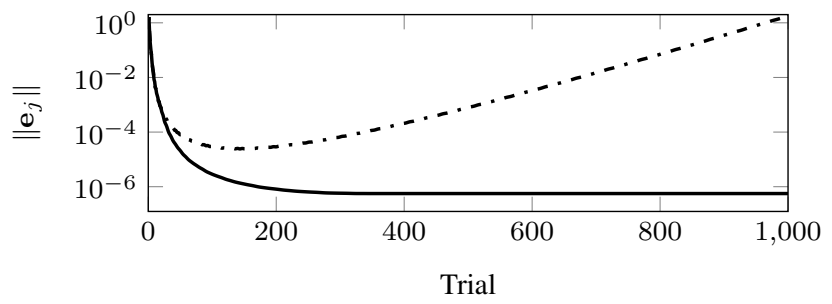

Fig. 9: Comparison: robust ILC (solid) and classical ILC (dashed)

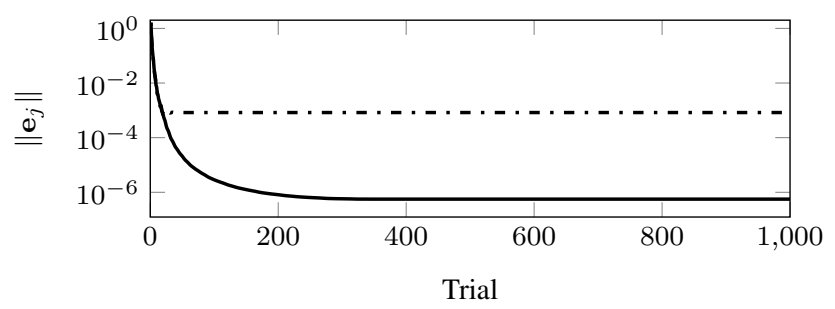

Fig. 10: Comparison: robust ILC (solid) and classical norm-optimal ILC with robust convergence condition (dashed) 
Fig. 9 shows the tracking errors of the robust ILC and classical norm-optimal ILC. It is seen that both approaches give very good performances during the first 100 trials. After that, the classical ILC error diverges, whereas the robust ILC still obtains monotonic convergence towards smaller tracking errors. The results again verify that the classical ILC can diverges as the number of trial increases.

Then, we increase $\mathbf{S}$ for the classical ILC in order to satisfy the robust monotonic convergence condition (8) and hence achieve monotonic convergence. After some tuning, the following gains are achieved: $(\mathbf{Q}, \mathbf{R}, \mathbf{S})=\left(1,10^{-5}, 10^{-3}\right) \mathbf{I}$. Accordingly, we implement the classical ILC algorithm with the updated gains. The results are then also compared to the robust design, which has the gains $(\mathbf{Q}, \mathbf{R}, \mathbf{S})=\left(1,10^{-5}, 0\right) \mathbf{I}$, shown in Fig. 10. The figure shows that the classical ILC accounting for the robust convergence condition can obtain monotonic convergence. However, the performance of the robust ILC design is significantly better. This can be explained by the fact that $\mathbf{S}=0$ in the robust ILC, while $\mathbf{S} \neq 0$ in the classical norm-optimal ILC.

\section{CONCLUSION}

The main contribution of this paper is a robust ILC design that can guarantee monotonic convergence in the presence of additive model uncertainty. The proposed robust ILC design approach corresponds to a convex optimization problem that can be solved efficiently. In addition, linear input constraints can be imposed. An interpretation of the robust ILC approach as an adaptive norm-optimal ILC with trialvarying learning gains is given. Detailed analyses and comparisons between the proposed robust ILC and classical norm-optimal ILC are presented together with illustrative simulations. These results show the benefits of the robust ILC approach in monotonic convergence and better performance.

\section{APPENDIX}

\section{A. Proof of Theorem 3.1}

Consider the following equivalent problem of (11):

$$
\begin{array}{ll}
\underset{\mathbf{u}_{j+1}}{\operatorname{minimize}} & \gamma+\left\|\mathbf{u}_{j+1}-\mathbf{u}_{j}\right\|_{\mathbf{R}}^{2}+\left\|\mathbf{u}_{j+1}\right\|_{\mathbf{S}}^{2} \\
\text { subject to } & \left\|\hat{\mathbf{e}}_{j+1}-\boldsymbol{\Delta} \mathbf{W}\left(\mathbf{u}_{j+1}-\mathbf{u}_{j}\right)\right\|_{\mathbf{Q}}^{2} \leq \gamma, \forall \boldsymbol{\Delta}:\|\boldsymbol{\Delta}\|<1 .
\end{array}
$$

The constraint is rewritten as

$$
\left\|\mathbf{f}_{j+1}-\mathbf{L} \boldsymbol{\Delta} \mathbf{v}_{j+1}\right\|^{2} \leq 1, \quad \forall \boldsymbol{\Delta}:\|\boldsymbol{\Delta}\|<1,
$$

where $\mathbf{Q}=\mathbf{L}^{T} \mathbf{L}, \mathbf{f}_{j+1}=\gamma^{-0.5} \mathbf{L} \hat{\mathbf{e}}_{j+1}, \mathbf{v}_{j+1}=\gamma^{-0.5} \mathbf{W}\left(\mathbf{u}_{j+1}-\right.$ $\left.\mathbf{u}_{j}\right)$. Then we denote $\mathbf{F}_{j+1}=\left[\begin{array}{llll}\mathbf{f}_{j+1} & \mathbf{0}_{N \times 1} & \cdots & \mathbf{0}_{N \times 1}\end{array}\right]$ and $\mathbf{V}_{j+1}=\left[\begin{array}{llll}\mathbf{v}_{j+1} & \mathbf{0}_{N \times 1} & \cdots & \mathbf{0}_{N \times 1}\end{array}\right]$, where $\mathbf{0}_{N \times 1}$ is a zero column vector and $\mathbf{F}_{j+1}, \mathbf{V}_{j+1} \in \mathbb{R}^{N \times N}$. Consequently, (28) is given by

$$
\bar{\sigma}\left(\mathbf{F}_{j+1}-\mathbf{L} \boldsymbol{\Delta} \mathbf{V}_{j+1}\right) \leq 1, \quad \forall \boldsymbol{\Delta}: \bar{\sigma}(\boldsymbol{\Delta})<1,
$$

where $\bar{\sigma}($.$) is the largest singular value.$

Next, we apply the results from the structured singular value theory, namely $\mu$-synthesis [20]. Consider the following linear fractional transformations (LFTs):

$\mathcal{F}\left(\mathbf{M}_{j+1}, \boldsymbol{\Delta}\right)=\mathbf{F}_{j+1}-\mathbf{L} \boldsymbol{\Delta} \mathbf{V}_{j+1}$, with $\mathbf{M}_{j+1}=\left[\begin{array}{cc}\mathbf{F}_{j+1} & -\mathbf{L} \\ \mathbf{V}_{j+1} & \mathbf{0}\end{array}\right]$,

and define a new structure $\hat{\Delta}$ by

$$
\hat{\boldsymbol{\Delta}}=\left\{\left[\begin{array}{cc}
\boldsymbol{\Delta}_{p} & \mathbf{0} \\
\mathbf{0} & \boldsymbol{\Delta}
\end{array}\right]: \boldsymbol{\Delta} \in \mathcal{B}_{\boldsymbol{\Delta}}^{\mathrm{o}}, \boldsymbol{\Delta}_{p} \in \mathcal{B}_{\boldsymbol{\Delta}}^{\mathrm{o}}\right\} .
$$

Denote the structured singular value with respect to $\hat{\Delta}$ as $\mu_{\hat{\Delta}}$. The main loop theorem (see Theorem 10.6 in [20]) shows that

$$
\sup _{\boldsymbol{\Delta} \in \mathcal{B}_{\boldsymbol{\Delta}}^{\circ}} \bar{\sigma}\left(\mathcal{F}\left(\mathbf{M}_{j+1}, \boldsymbol{\Delta}\right)\right) \leq 1 \Longleftrightarrow \mu_{\hat{\boldsymbol{\Delta}}}\left(\mathbf{M}_{j+1}\right) \leq 1 .
$$

Note that $\mu_{\boldsymbol{\Delta}_{p}}()=.\bar{\sigma}($.$) for unstructured \boldsymbol{\Delta}_{p}$. On the other hand, $\mu_{\hat{\boldsymbol{\Delta}}}\left(\mathbf{M}_{j+1}\right)=\inf _{\mathbf{D}_{j+1}} \bar{\sigma}\left(\mathbf{D}_{j+1} \mathbf{M}_{j+1} \mathbf{D}_{j+1}^{-1}\right)$, where $\mathbf{D}_{j+1}$ is any nonsingular matrix such that $\mathbf{D}_{j+1} \hat{\boldsymbol{\Delta}}=\hat{\boldsymbol{\Delta}} \mathbf{D}_{j+1}$ [20]. Set $\mathbf{D}_{j+1}=\operatorname{diag}\left(\mathbf{I}, d_{j+1} \mathbf{I}\right), d_{j+1}>0$, then

$$
\begin{aligned}
\mu_{\hat{\boldsymbol{\Delta}}}\left(\mathbf{M}_{j+1}\right) \leq 1 & \Leftrightarrow \exists \mathbf{D}_{j+1} \succ 0: \bar{\sigma}\left(\mathbf{D}_{j+1} \mathbf{M}_{j+1} \mathbf{D}_{j+1}^{-1}\right) \leq 1 \\
& \Leftrightarrow \exists \mathbf{D}_{j+1} \succ 0: \mathbf{M}_{j+1}^{T} \mathbf{D}_{j+1}^{2} \mathbf{M}_{j+1}-\mathbf{D}_{j+1}^{2} \preceq 0 .
\end{aligned}
$$

The last inequality is equivalent to the existence of a scalar $d_{j+1} \geq 0$ subject to

$$
\left[\begin{array}{cc}
\mathbf{I}-\left(\mathbf{F}_{j+1}^{T} \mathbf{F}_{j+1}+d_{j+1}^{2} \mathbf{V}_{j+1}^{T} \mathbf{V}_{j+1}\right) & \mathbf{F}_{j+1}^{T} \mathbf{L} \\
\mathbf{L}^{T} \mathbf{F}_{j+1} & d_{j+1}^{2} \mathbf{I}-\mathbf{L}^{T} \mathbf{L}
\end{array}\right] \succeq 0 .
$$

Using the Schur complement [19], (35) is equivalent to

$$
\begin{array}{r}
\left\{\begin{array}{r}
d_{j+1}^{2} \mathbf{I}-\mathbf{L}^{T} \mathbf{L} \succeq 0 ; \quad \mathbf{L}^{T} \mathbf{F}_{j+1} \in \mathcal{R}\left(d_{j+1}^{2} \mathbf{I}-\mathbf{L}^{T} \mathbf{L}\right) \\
\mathbf{I}-\left(\mathbf{F}_{j+1}^{T} \mathbf{F}_{j+1}+d_{j+1}^{2} \mathbf{V}_{j+1}^{T} \mathbf{V}_{j+1}\right) \\
-\mathbf{F}_{j+1}^{T} \mathbf{L}\left(d_{j+1}^{2} \mathbf{I}-\mathbf{L}^{T} \mathbf{L}\right)^{\dagger} \mathbf{L}^{T} \mathbf{F}_{j+1} \succeq 0
\end{array}\right. \\
\Leftrightarrow\left\{\begin{array}{r}
d_{j+1}^{2} \mathbf{I}-\mathbf{Q} \succeq 0 ; \mathbf{Q} \hat{\mathbf{e}}_{j+1} \in \mathcal{R}\left(d_{j+1}^{2} \mathbf{I}-\mathbf{Q}\right) \\
d_{j+1}^{2}\left\|\mathbf{W}\left(\mathbf{u}_{j+1}-\mathbf{u}_{j}\right)\right\|^{2}+\hat{\mathbf{e}}_{j+1}^{T}\left(\mathbf{Q}^{-1}-d_{j+1}^{-2} \mathbf{I}\right)^{\dagger} \hat{\mathbf{e}}_{j+1} \leq \gamma,
\end{array}\right.
\end{array}
$$

where the notation $B \in \mathcal{R}(A)$ means that all columns of $B$ are in the range of $A$. Moreover, the last inequality is obtained from the following equalities: $\mathbf{Q}+\mathbf{Q}\left(d_{j+1}^{2} \mathbf{I}-\mathbf{Q}\right)^{\dagger} \mathbf{Q}=\left(d_{j+1}^{2} \mathbf{I}-\mathbf{Q}\right)^{\dagger} d_{j+1}^{2} \mathbf{Q}$ and $d_{j+1}^{2} \mathbf{I}-\mathbf{Q}=\left(d_{j+1}^{2} \mathbf{Q}\right)\left(\mathbf{Q}^{-1}-d_{j+1}^{-2} \mathbf{I}\right)$. As a result, by denoting $\lambda_{j+1}=d_{j+1}^{2}$, this yields Theorem 3.1.

\section{B. Convergence proof with symmetric matrix $\mathbf{Q}$}

Consider the problem (12), if $\mathbf{u}_{j+1}=\mathbf{u}_{j}$ then the solution of $\lambda_{j+1}$ is given by $\bar{\lambda}_{j+1}=+\infty$ or $\bar{\lambda}_{j+1}^{-1}=0$. Moreover, we have $J_{\text {dual }}\left(\mathbf{u}_{j+1}, \lambda_{j+1}\right) \leq J_{\text {dual }}\left(\mathbf{u}_{j}, \bar{\lambda}_{j+1}\right)$, yielding

$$
\begin{aligned}
J_{\text {dual }}\left(\mathbf{u}_{j+1}, \lambda_{j+1}\right) & \leq \mathbf{e}_{j}^{T}\left(\mathbf{Q}^{-1}-\bar{\lambda}_{j+1}^{-1} \mathbf{I}\right)^{\dagger} \mathbf{e}_{j}+\left\|\mathbf{u}_{j}\right\|_{\mathbf{S}}^{2} \\
& \leq\left\|\mathbf{e}_{j}\right\|_{\mathbf{Q}}^{2}+\left\|\mathbf{u}_{j}\right\|_{\mathbf{S}}^{2}
\end{aligned}
$$

The equality happens only when $\mathbf{u}_{j+1}=\mathbf{u}_{j}$ since the objective function in (13b) is a strictly convex function [19]. In addition, $\lambda_{j+1}$ is the solution of the dual problem of the worst-case problem thus

$$
\left\|\mathbf{e}_{j+1}\right\|_{\mathbf{Q}}^{2}+\left\|\mathbf{u}_{j+1}\right\|_{\mathbf{S}}^{2} \leq J\left(\mathbf{u}_{j+1}, \boldsymbol{\Delta}\right) \leq J_{\text {dual }}\left(\mathbf{u}_{j+1}, \lambda_{j+1}\right) .
$$

Consequently, the following relationship is obtained

$$
\left\|\mathbf{e}_{j+1}\right\|_{\mathbf{Q}}^{2}+\left\|\mathbf{u}_{k+1}\right\|_{\mathbf{S}}^{2} \leq J_{\text {dual }}\left(\mathbf{u}_{j+1}, \lambda_{j+1}\right) \leq\left\|\mathbf{e}_{j}\right\|_{\mathbf{Q}}^{2}+\left\|\mathbf{u}_{j}\right\|_{\mathbf{S}}^{2},
$$

and the inequalities are strict unless $\mathbf{u}_{j+1}=\mathbf{u}_{j}$.

\section{ACKNOWLEDGMENTS}

This work was supported by the European Commission under the EU Framework 7 funded Marie Curie Initial Training Network (ITN) IMESCON (grant no. 264672). This work also benefits from KU Leuven-BOF PFV/10/002 Cente of Excellence: Optimization in Engineering (OPTEC), and from the Belgian Network Dynamical Systems, Control and Optimization (DYSCO), initiated by the Belgian Science Policy Office. Goele Pipeleers is Postdoctoral Fellow of the Research Foundation Flanders (FWO). 


\section{REFERENCES}

[1] D. A. Bristow, M. Tharayil, and A. G. Alleyne, "A survey of iterative learning control: a learning based method for high-performance tracking control," IEEE Control Systems Magazine, vol. 26, pp. 96-114, June 2006.

[2] T. J. Harte, J. Hatonen, and D. H. Owens, "Discrete-time inverse modelbased iterative learning control: stability, monotonicity and robustness," International Journal of Control, vol. 78, no. 8, pp. 577-586, 2005.

[3] T. Donkers, J. van de Wijdeven, and O. Bosgra, "Robustness against model uncertainties of norm optimal iterative learning control," in Proceedings of the American Control Conference, 2008.

[4] A. Haber, R. Fraanje, and M. Verhaegen, "Linear computational complexity robust ILC for lifted systems," Automatica, vol. 48, no. 6, pp. $1102-1110,2012$

[5] B. Cichy, K. Galkowski, and E. Rogers, "2D systems based robust iterative learning control using noncausal finite-time interval data," Systems \& Control Letters, vol. 64, no. 0, pp. 36 - 42, 2014.

[6] D. Owens and S. Daley, "Robust gradient iterative learning control: time and frequency domain conditions," International Journal of Modelling, Identification and Control, vol. 4, no. 4, pp. 315-322, 2008.

[7] K. Moore, Y. Chen, and H.-S. Ahn, "Algebraic $\mathcal{H}_{\infty}$ design of higherorder iterative learning controllers," in Proceedings of the IEEE International Symposium on Intelligent Control, pp. 1207 -1212, june 2005.

[8] D. Bristow and A. Alleyne, "Monotonic convergence of iterative learning control for uncertain systems using a time-varying filter," Automatic Control, IEEE Transactions on, vol. 53, pp. 582-585, march 2008.

[9] M. Q. Phan, R. W. Longman, B. Panomruttanarug, and S. C. Lee, "Robustification of iterative learning control and repetitive control by averaging," International Journal of Control, vol. 86, no. 5, pp. 855868,2013

[10] H.-S. Ahn, K. Moore, and Y. Chen, "Monotonic convergent iterative learning controller design based on interval model conversion," IEEE Transactions on Automatic Control, vol. 51, pp. 366-371, feb. 2006.

[11] D. Roover, "Synthesis of a robust iterative learning controller using an $\mathcal{H}_{\infty}$ approach," in Proceedings of the 35th IEEE Conference on Decision and Control, vol. 3, pp. 3044-3049, dec 1996.

[12] K. L. Moore, H.-S. Ahn, and Y. Q. Chen, "Iteration domain $\mathcal{H}_{\infty}$ optimal iterative learning controller design," International Journal of Robust and Nonlinear Control, vol. 18, no. 10, pp. 1001-1017, 2008.

[13] S. Skogestad and I. Postlethwaite, Multivariable feedback control: analysis and design. John Wiley, 2005.

[14] D. H. Nguyen and D. Banjerdpongchai, "A convex optimization approach to robust iterative learning control for linear systems with timevarying parametric uncertainties," Automatica, vol. 47, no. 9, pp. 20392043, 2011.

[15] J. J. M. van de Wijdeven, M. C. F. Donkers, and O. H. Bosgra, "Iterative learning control for uncertain systems: Noncausal finite time interval robust control design," International Journal of Robust and Nonlinear Control, vol. 21, no. 14, pp. 1645-1666, 2011.

[16] K. Barton and A. Alleyne, "A norm optimal approach to time-varying ILC with application to a multi-axis robotic testbed," IEEE Transactions on Control Systems Technology, vol. 19, pp. 166-180, jan. 2011.

[17] M. Norrlöf and S. Gunnarsson, "Time and frequency domain convergence properties in iterative learning control," International Journal of Control, vol. 75, no. 14, pp. 1114-1126, 2002.

[18] S. Gunnarsson and Mikael Norrlöf, "On the design of ILC algorithms using optimization," Automatica, vol. 37, no. 12, pp. 2011-2016, 2001.

[19] S. Boyd and L. Vandenberghe, Convex Optimization. Cambridge University Press, 2004.

[20] K. Zhou and J. C. Doyle, Essentials of Robust Control. Upper Saddle River, NJ: Prentice-Hall, 1998. 